\title{
K-band magnitude of the red clump as a distance indicator ${ }^{\star}$
}

\author{
V. Van Helshoecht and M. A. T. Groenewegen
}

\author{
Instituut voor Sterrenkunde, KU Leuven, Celestijnenlaan 200D, 3001 Leuven, Belgium \\ e-mail: veerle@ster.kuleuven. be
}

Received 18 January 2005 / Accepted 21 September 2006

ABSTRACT

\begin{abstract}
We have investigated how the $K$-band magnitude of the red clump $\left[M_{K}(R C)\right]$ depends on age and metallicity, using 2MASS infrared data for a sample of 24 open clusters with known distances. We show that a constant value of $M_{K}(R C)=-1.57 \pm 0.05$ is a reasonable assumption to use in distance determinations for clusters with metallicity between -0.5 and +0.4 dex and age between $10^{8.5}$ and $10^{9.9}$ years.
\end{abstract}

Key words. open clusters and associations: general - stars: horizontal-branch - stars: distances

\section{Introduction}

Besides the main sequence, another highly populated region in the color-magnitude diagram [CMD] of several open clusters can be identified and is called the red clump [RC]. This is the horizontal branch of an open cluster, that is slightly clumped together. The red clump is made up of helium-burning giant stars, just like a normal horizontal branch. The RC can be used as a standard candle, with the advantage that it is easily recognizable in the CMD of most open clusters. Once its absolute magnitude is obtained, one can determine the distance by knowing the apparent magnitude of the red clump. There is still no unanimity in the literature about the appropriate treatment of possible age $[\tau]$ and metallicity $[[\mathrm{Fe} / \mathrm{H}]]$ dependences, whether on the $I$-band or the $K$-band absolute magnitude of the red clump.

The dependence of the $I$-band magnitude on the RC stars was extensively studied in the past from an observational point of view. Udalski (1998) has analyzed red clump stars in 15 Magellanic cloud clusters and concluded that the absolute $I$-band magnitude of the $\mathrm{RC}\left[M_{I}(R C)\right]$ in these clusters does not depend on age for an intermediate-age population (2-10 Gyr).

In Paczyński \& Stanek (1998) and Stanek \& Garnavich (1998) the $M_{I}$ of solar neighborhood RC stars were compared with red clump stars in Baade's window and in three fields in $M 31$, respectively. Little or no variation in $M_{I}$ with color and hence no significant variation with metallicity, was found. Sarajedini (1999) presented observations of 8 open clusters, concluding that $M_{I}(R C)$ is less sensitive to metal abundance than $M_{V}(R C)$, but that the dependence on age and metallicity is still not negligible. Afterwards, Udalski (2000) also stated, based on 284 nearby red giant stars with high-quality spectra, that the $M_{I}$ of red clump stars weakly depends on metallicity $\left(\approx 0.13 \mathrm{mag} \mathrm{dex}^{-1}\right)$. Later observational studies of Zhao et al. (2001) and Kubiak et al. (2002) agree with that conclusion. Theoretical models from Girardi \& Salaris (2001) also show a dependence in the $I$-band, predicting that an older cluster with higher metallicity has a fainter red clump.

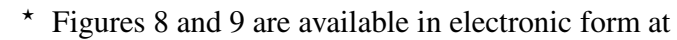
http: //www . aanda.org
There is also a controversy in the $K$-band. Theory predicts a small dependence on age and $[\mathrm{Fe} / \mathrm{H}]$ (Salaris \& Girardi 2002) see Figs. 6 and 7, while observational results show that the dependence appears negligible in most cases. The analysis of Alves (2000) relies upon the $K$-band luminosity, because it is less sensitive to interstellar extinction. For a sample of $238 \mathrm{RC}$ stars in the solar neighborhood, he finds a mean value of $M_{K}=-1.61 \pm 0.03$ and concludes that there is no significant correlation between $M_{K}$ and metallicity. Similar to Sarajedini (1999) in the I-band, Grocholski \& Sarajedini (2002) investigated the dependence for the $K$-band RC. Based on 14 open clusters, they draw the conclusion that for clusters having $-0.5 \leq$ $[\mathrm{Fe} / \mathrm{H}] \leq 0.0$ and $10^{9.2} \leq \tau \leq 10^{9.9}$ years, one can simply use $\left\langle M_{K}(R C)\right\rangle=-1.61 \pm 0.04$.

Later Percival \& Salaris (2003) redetermined the distance moduli to 9 clusters, applying their empirical main-sequence fitting [MSF] method. A shift in de-reddened distance moduli compared to the determinations by Twarog et al. (1997) can be seen. Since Grocholski \& Sarajedini (2002) used the latter data set, this shift results in a mean difference in $M_{K}(R C)$ between values in both articles. If we only focus on the 5 common clusters, we see that the de-reddened distance moduli calculated by Percival \& Salaris (2003) are on average +0.06 mag smaller than the one of Twarog et al. (1997), so the $M_{K}(R C)$ determined by Grocholski \& Sarajedini (2002) is on average 0.06 mag brighter than the value stated in Percival \& Salaris (2003) for the same cluster. The authors of this article find complete agreement between the observations and the theory of Girardi et al. (2000). We note that the theoretical models of Salaris \& Girardi (2002) are based on Girardi et al. (2000).

Pietrzyński et al. (2003) have also investigated the dependence of the mean $K, J$, and $I$ absolute magnitudes of red clump stars on metallicity and age, as a part of their ongoing Araucaria Project to improve stellar distance indicators. They took deep near-infrared $J K$ imaging of several fields in LMC, SMC, and the Carina and Fornax dwarf galaxies and made a comparison between the extinction-corrected $K$-band red clump star magnitudes and some other stellar distance indicators, particularly the tip of the red giant-branch magnitude, the mean RR Lyrae star $V$-band magnitude, and the mean $K$-band magnitude of Cepheid 
variables at a period of 10 days. That comparison strongly suggests that the red clump-star's absolute $K$-band magnitude has a very low dependence (if any) on $[\mathrm{Fe} / \mathrm{H}]$ over the broad range of metallicities covered by their target galaxies. They conclude that the mean $K$-band magnitude of red clump stars is an excellent distance indicator with small (if any) population corrections to be applied over a wide range in metallicity and age.

We focus on the dependence of $M[R C]$ in the $K$-band by extending the cluster sample of 14 in Grocholski \& Sarajedini (2002) to 24 clusters. A larger sample could offer more clarity on this issue, leading to more accurate distance determinations. Moreover, we use the 2MASS All Sky Release instead of the 2nd incremental data release used by Grocholski \& Sarajedini (2002). Preliminary results are published in Van Helshoecht \& Groenewegen (2006). In Sect. 2 both cluster data and data of individual stars will be discussed. How the data was reduced can be found in Sect. 3, and in Sect. 4 we explain our results. Finally a discussion of this issue follows in Sect. 5.

\section{The data}

We made a selection of open clusters, based on their age, availability of consistent data, and the presence of a clear red clump in its CMD in our research. Data on these 24 selected clusters can be found in Table 3. This table is divided into three parts. In Cols. 2 and 3, the reddening and distance moduli are listed. This information is needed, together with apparent magnitudes of the individual cluster stars, to determine the $M_{K}(R C)$ of a cluster. In Cols. 4 to 6 one can find the metallicity and ages of the clusters. These data are required to investigate how the $K$-band magnitudes of the red clump depend on age and metallicity. In the last two columns, the $M_{K}(R C)$ of each cluster resulting from our study, together with its error, can be found.

According to the ages published by Chen et al. (2003), all selected clusters are older than $10^{8.5}$ years. Younger clusters have fewer or no cluster stars in the evolutionary phase of helium burning and may have an unclear or even no red clump. When the CMD is strongly contaminated with field stars or poorly filled, the RC might also be difficult to recognize. Clusters without a clear red clump were excluded.

For the cluster reddenings $(E(B-V))$ and distance moduli $(m-M)$, we used values of Twarog et al. (1997), who made a consistent list of data for 76 open clusters. They list the reddening for both main sequence stars and red giant stars, and the latter was adopted here. The reddening of NGC 6819 found by Twarog et al. (1997) is much higher than other literature values for that cluster. Just like Grocholski \& Sarajedini (2002), we decided to adopt the reddening of Sarajedini (1999) for that cluster. For NGC 2477 the giants reddening is not a constant in the cluster according to Twarog et al. (1997), and 90\% of the reddening for main sequence stars is adopted, as suggested in Grocholski \& Sarajedini (2002).

All values of the distance moduli of the cluster sample were derived with the same technique-MSF-used by Twarog et al. (1997). This means we excluded NGC 2539, even though it was included in Grocholski \& Sarajedini (2002).

As for the reddening, we decided to adopt the distance modulus of Sarajedini (1999) for NGC 6819, because calculations of these parameters are connected. Except for this one case, we decided not to include moduli found in other articles, even if MSF is used. For example, after comparing MSF distances of Bragaglia \& Tosi (2006) with those of Twarog et al. (1997), we found a mean difference in the values of the distance of only 0.01 with a standard deviation of $0.61 \mathrm{kpc}$, so there are no systematic

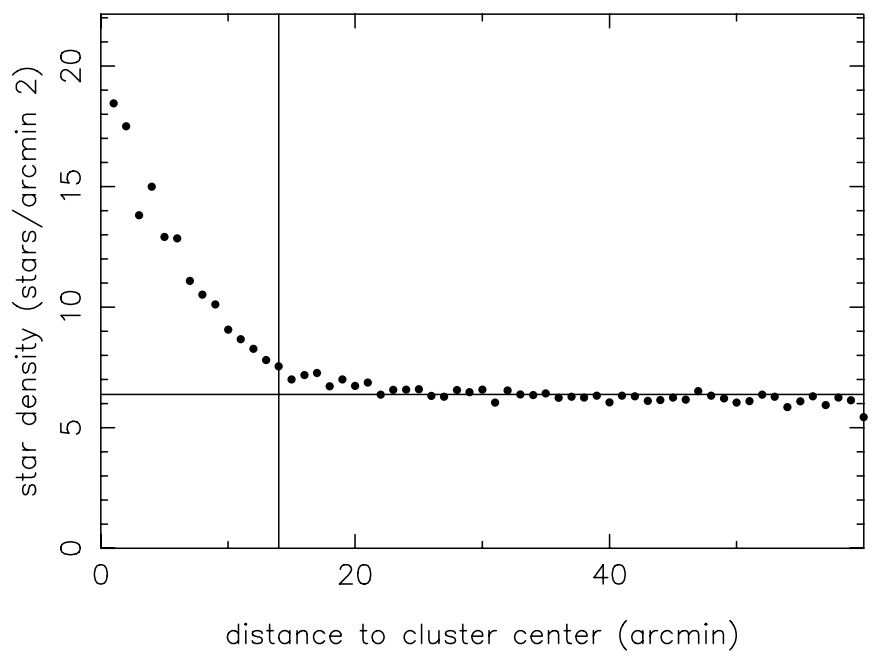

Fig. 1. An example of how membership was determined, for NGC 7789. The star density is plotted as a function of the distance to the cluster center for an area of $60 \mathrm{arcmin}$ around the center. We define the radius as the distance to the cluster center where the star density is $120 \%$ of the field density.

differences between both investigations. Another case is the distances determined by Percival \& Salaris (2003). There we do find a systematic difference, a mean difference of $0.07 \mathrm{kpc}$ and a mean deviation of $0.12 \mathrm{kpc}$. As can be seen, we find that the consistency of the distance related parameters very important, since we aim to calibrate $M_{K}(R C)$ as a distance indicator.

We adopted the metallicities $([\mathrm{Fe} / \mathrm{H}])$ of Gratton (2000), who constructed a consistent $[\mathrm{Fe} / \mathrm{H}]$ scale for old open clusters. NGC 2099 is the only cluster under investigation that is not listed there, so we based it on the DDO $[\mathrm{Fe} / \mathrm{H}]$ published in Piatti et al. (1995) for this cluster. Then we applied the same correction as used and described by Gratton (2000) to the value of $[\mathrm{Fe} / \mathrm{H}]$ and its error. For NGC 2099, this results in $[\mathrm{Fe} / \mathrm{H}]=+0.27 \pm 0.15$.

Besides the 14 open clusters, Grocholski \& Sarajedini (2002) investigated two globular ones to obtain knowledge of $M_{K}(R C)$ outside the ranges in age and metallicity mentioned above. We also consider 47 Tuc and NGC 362 in our research, using data of (Grocholski \& Sarajedini 2002; see also Table 3).

Data for the individual stars is provided by the 2 Micron All Sky Survey $[2 M A S S]^{1}$. This recent project surveyed the entire sky at near-infrared wavelengths between 1997 and 2001. It observed in the $J[1.25 \mu \mathrm{m}], H[1.65 \mu \mathrm{m}]$, and $K$-short $\left[K_{\mathrm{S}}=\right.$ $2.17 \mu \mathrm{m}]$ bands. Grocholski \& Sarajedini (2002) used the second incremental data release, while we use the All Sky Survey. A read-flag is included in the catalog to indicate how the photometry of a source was measured. We exclude stars with a read-flag of zero in any band, because then it was not detected and the given magnitude is just an upper limit.

\section{Data analysis}

The same data reduction protocol was followed for each cluster as is illustrated here for NGC 7789. The adopted radius of the cluster was based on the star density in the center's neighborhood, see Fig. 1. As one can see, there is a higher star density near the cluster center, while further out a quasi-constant density of the field is measured. A cluster radius in the plane of the sky is determined based on this type of figure; we define the radius as

\footnotetext{
${ }^{1}$ http://www.ipac. caltech.edu/2mass
} 


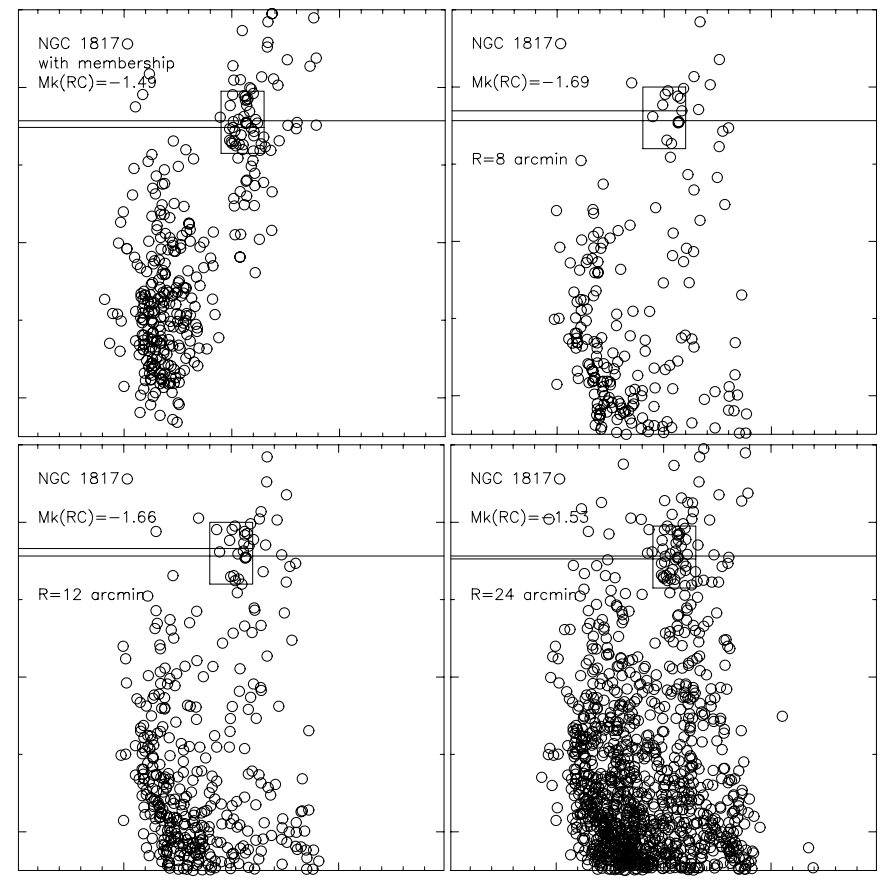

Fig. 2. Cluster NGC 1817. Top left: CMD build up with stars within the radius stated by WEBDA. Top right: CMD build up with probable members, also according WEBDA. Bottom: CMD build up with stars within a certain radius, resp. 12 and 24 arcmin.

the distance to the cluster center where the star density is $120 \%$ of the field density.

After visual inspection, the radius was changed manually in some cases, so that the radius was chosen such that it provides the clearest red clump in the CMD. This approach may result in a radius that is larger than the one stated in $\mathrm{WEBDA}^{2}$ for that particular cluster. Still our choice is confirmed by comparing some CMDs with their counterpart based on membership probabilities.

We found 8 clusters out of our sample that have membership determinations (see WEBDA and references therein) and more than 5 stars contributing to the RC. All stars with a probability $>0.3$ were counted as cluster stars, and then exactly the same data analysis procedure was applied to make a comparison. The difference in $M_{K}(R C)$ between both types of analysis (1 with all stars within the cluster radius based on star density, the other study based on stars that are more than $30 \%$ certain to be a cluster member) is less than $0.02 \mathrm{mag}$ in 7 out of the 8 cases. This is without doubt inside the error margin. We found large difference for only 1 cluster, NGC 1817 . This difference is not caused by a cluster radius that was chosen too large. In the top left graph in Fig. 2 the result of the study can be seen that only includes stars that have a more than $30 \%$ probability of being a member of the cluster. The other graphs show resulting CMDs of studies, including stars within the mentioned radius. The radius of NGC 1817 listed by WEBDA is 8 arcmin, the radius found by our delimiting procedure described above is 12 arcmin. Comparing those graphs with the membership-based $\mathrm{CMD}$, we can conclude that a radius, larger than the one stated by WEBDA, is allowed in some cases.

We adopted the results of the study based on membership probabilities for the 8 clusters: NGC 188, NGC 752, NGC 1817, NGC 2099, NGC 2420, NGC 2682, NGC 3680, NGC 7789. In

\footnotetext{
${ }^{2}$ http://obswww. unige.ch/webda
}

Table 1. Comparison of the radii with WEBDA.

\begin{tabular}{lcccc}
\hline \hline Cluster & $\begin{array}{c}\text { Radius } \\
\text { this study } \\
\text { arcmin }\end{array}$ & $\begin{array}{c}\text { Radius } \\
\text { WEBDA } \\
\text { arcmin }\end{array}$ & $\begin{array}{c}\text { Field } \\
\text { density } \\
\text { arcmin }^{-2}\end{array}$ & $\begin{array}{c}\text { \% Real } \\
\text { cluster stars }\end{array}$ \\
\hline BE 39 & 6.0 & 3.5 & 3 & 49 \\
IC 4651 & 6.5 & 5.0 & 15 & 8 \\
MEL 66 & 10.0 & 7.0 & 3 & 43 \\
NGC 2204 & 8.0 & 5.0 & 2 & 49 \\
NGC 2243 & 8.0 & 2.5 & 2 & 57 \\
NGC 2360 & 10.0 & 6.5 & 7 & 15 \\
NGC 2477 & 15.0 & 7.5 & 7 & 38 \\
NGC 2506 & 13.0 & 6.0 & 3 & 54 \\
NGC 2527 & 18.0 & 5.0 & 9 & 3 \\
NGC 3960 & 3.0 & 2.5 & 10 & 56 \\
NGC 5822 & 17.5 & 17.5 & 21 & 0 \\
NGC 6134 & 15.0 & 3.0 & 34 & 1 \\
NGC 6633 & 30.0 & 10.0 & 11 & 4 \\
NGC 6791 & 3.0 & 5.0 & 6 & 81 \\
NGC 6819 & 12.0 & 2.5 & 7 & 42 \\
TOM 2 & 4.0 & 1.5 & 7 & 57 \\
\hline
\end{tabular}

Table 1 the radius, determined using the star density, can be compared with the radius tabulated by WEBDA for the remaining 16 clusters.

Within these radii cluster stars are present, along with the field stars. We can estimate the percentage of field stars by extrapolating the field density to this region. The estimated field density expressed in stars per square arc minutes and an estimation for the percentage of cluster stars for each cluster can also be found in Table 1. Although the percentages are near zero in some cases, we are still looking at the red clump of the cluster itself. When drawing the CMD of the cluster in the next step of the data analysis, we calculated the $K$-band absolute magnitude for each star. Because each star within the cluster radius is supposed to be a cluster star, we assumed that they are all at the same distance, i.e. the cluster distance. This assumption is wrong for the field stars within the radius, so their calculated $M_{K}$ will be incorrect. This causes only a minority of the field stars to contaminate the area in the CMD where the RC of the cluster is located.

With the so-called cluster stars, the cluster CMD is drawn. The apparent magnitudes $\left[m_{\mathrm{ob}}\right]$ of individual stars, observed by the 2MASS, are combined with the cluster reddening and distance, to calculate the absolute magnitudes. For that, we use values of Cardelli et al. (1989), in particular $R_{V}=3.1, A_{K}=$ $0.11 \cdot A_{V}$, and $A_{J}=0.28 \cdot A_{V}$. The 2MASS program, which our analysis is based on, provides data in the $K_{\mathrm{S}}$ band. Salaris $\&$ Girardi (2002) used the $K$ band of $2.2 \mu \mathrm{m}$ to calculate their theoretical models. To compare with their results and those of Grocholski \& Sarajedini (2002), we chose to convert the 2MASS magnitudes $M_{K_{\mathrm{S}}}$ of the all sky data release adopting the Bessell \& Brett (1988) (BB) system, as suggested by Carpenter (2001):

$M_{K}=\left(M_{K_{\mathrm{S}}}-(-0.044 \pm 0.003)\right)-(0.000 \pm 0.005)\left(M_{J}-M_{K}\right)$

Absolute magnitudes $\left[M_{K}\right]$ of all cluster members are plotted against color $\left[m_{J}-m_{K}\right]$ in the same CMD, see Fig. 3. Analog figures for the other clusters can be found in the Figs. 8 and 9.

Then a search routine was implemented to find the RC in the CMD, knowing that it is a highly populated spot and must be found at a brighter $M_{K}$ than the main sequence. A fixed-size box of $0.2 \mathrm{mag}$ in color and $0.8 \mathrm{mag}$ in magnitude is put around the $\mathrm{RC}$. In some cases, the box was replaced manually. An incorrect localization of the box could happen due to contamination 


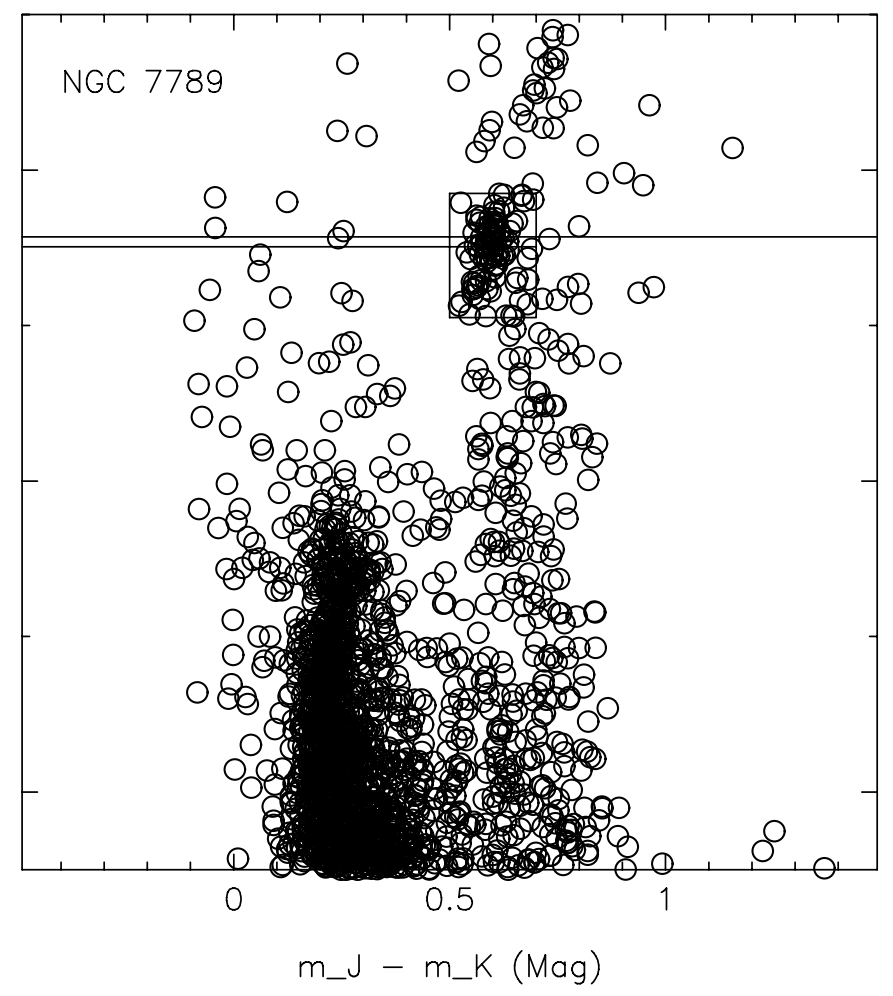

Fig. 3. CMD of cluster NGC 7789. The CMD of all clusters are shown in Figs. 8 and 9.

by field stars or, more importantly, if the position of the main sequence affects the search routine negatively.

Finally the median $M_{K}$ of the stars in the box is calculated. We prefer the median instead of the mean, in order to minimize the influence of stars in the box that are not red clump stars. Paczyński \& Stanek (1998) have calculated the galactocentric distance with the use of red clump stars. In their five color bins, analogous to our red clump box, they fitted the distribution with a function that exists of a linear term to describe the background and a Gaussian fit to the red clump star distribution itself. The amount of red clump stars in our boxes is very small, compared to the number in the bins. This is the reason this approach was not adopted in this research.

Two horizontal lines can be seen in the CMD. The half line, coming out of the RC box, is drawn at the median $M_{K}(R C)$ of that particular cluster. The other line represents the mean $M_{K}(R C)$ of the entire cluster sample.

The uncertainty in $M_{K}(R C)$ is calculated by combining the standard error in the mean in the box and the error in the $M_{K}(\star)$ of each individual star. Assuming that the error on the observed magnitude of a star can be neglected, $M_{K}(\star)$ is a combination of errors in $E(B-V)$ and $(m-M)_{V}$,

$\sigma^{2}\left(M_{K}(\star)\right)=\sigma^{2}(0.89 \cdot 3.1 \cdot E(B-V))+\sigma^{2}\left((m-M)_{V}\right)$.

Errors in the reddening and distance modulus are taken to be 0.02 and $0.1 \mathrm{mag}$, respectively. $M_{K}(R C)$, together with its uncertainty, is tabulated in Table 3. The error is in the range between 0.11 and $0.12 \mathrm{mag}$ for the clusters in this investigation, and the mean error is $0.11 \mathrm{mag}$. The major contribution to this error comes from the uncertainty in the distance modulus.
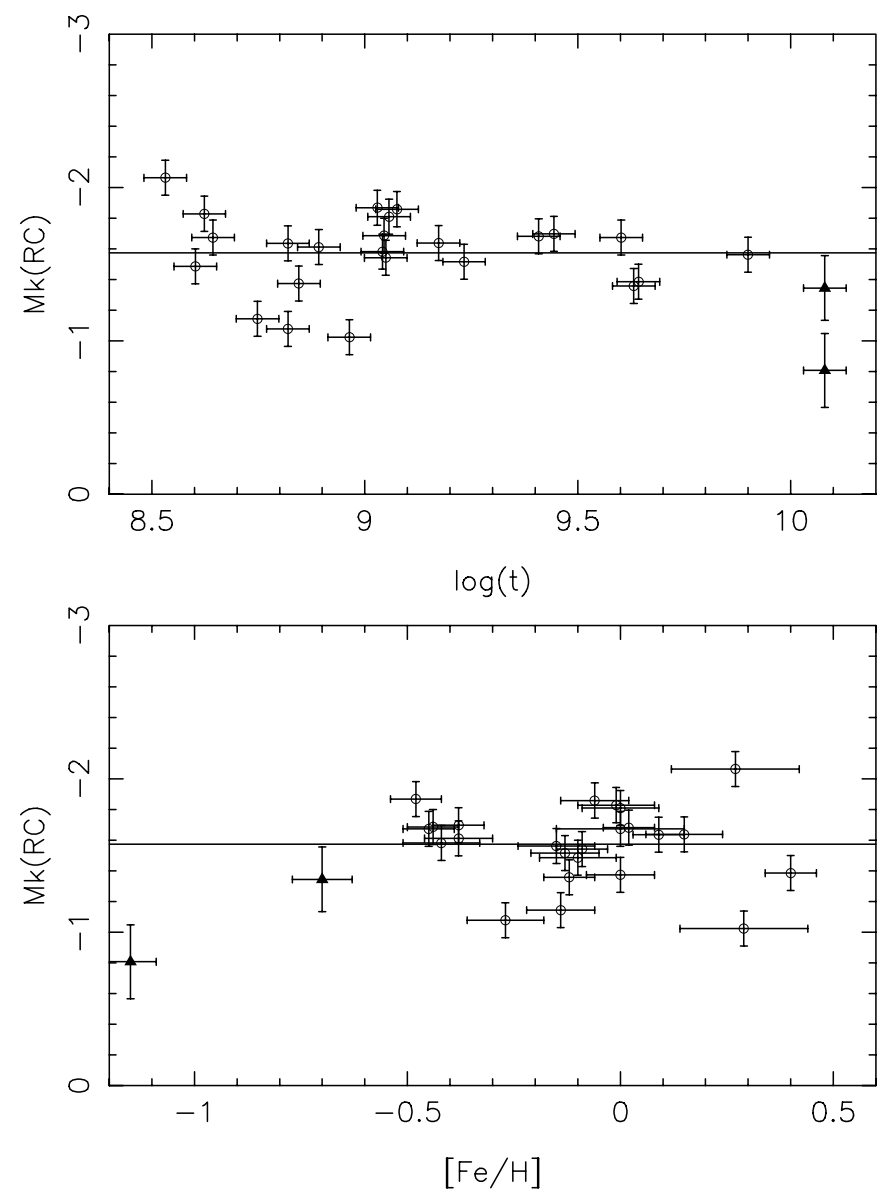

Fig. 4. Absolute $K$-magnitude dependence of the RC on age $(t)$ and metallicity $([\mathrm{Fe} / \mathrm{H}])$. The two triangles represent the globular clusters.
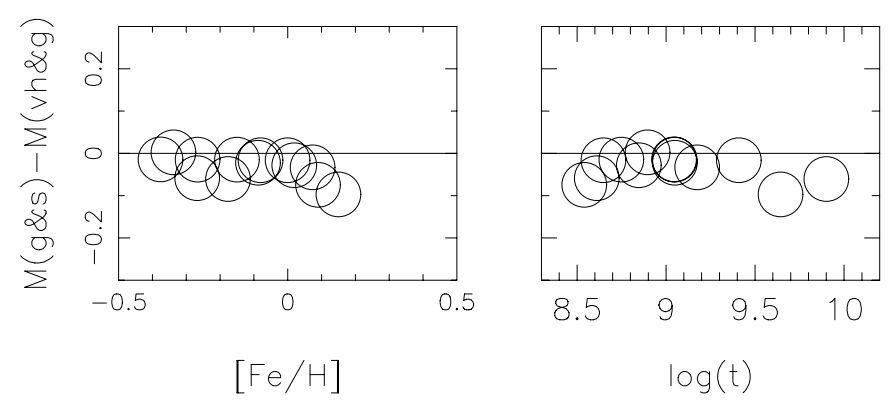

Fig. 5. Comparison of our results [vh\&g], based on the all-sky release (so without the use of membership), with Grocholski \& Sarajedini (2002) [g\&s]. The error bars are smaller than the the size of the symbols.

\section{Results}

We investigated the dependence of the $M_{K}(R C)$ of open clusters on age or metallicity and show our results in Fig. 4. Two older and metal poor globular clusters, NGC 362 and 47 Tuc are also shown. The mean $\left\langle M_{K}(R C)\right\rangle$ of the open cluster sample is $-1.57 \mathrm{mag}$. The standard deviation amounts to $0.25 \mathrm{mag}$. The mean deviation from $\left\langle M_{K}(R C)\right\rangle$ is estimated to be 0.19 mag.

Using the same technique as Grocholski \& Sarajedini (2002), it is surprising that our results are slightly shifted with respect to each other, as can be seen in Fig. 5 or in Table 2. In that figure we plotted the differences between the $M_{K}(R C)$ of Grocholski \& Sarajedini (2002) and our determined value for every cluster. Because the same distance moduli and 
Table 2. Comparison with Grocholski \& Sarajedini (2002), [G\&S].

\begin{tabular}{lccccc}
\hline \hline Name & $\begin{array}{c}M_{K}(R C) \\
\text { members }\end{array}$ & $\begin{array}{c}M_{K}(R C) \\
{[\mathrm{VH} \& \mathrm{G}]}\end{array}$ & $\begin{array}{c}\sigma\left(M_{K}(R C)\right) \\
{[\mathrm{VH} \& \mathrm{G}]}\end{array}$ & $\begin{array}{c}M_{K}(R C) \\
{[\mathrm{G} \& \mathrm{~S}]}\end{array}$ & $\begin{array}{c}\sigma\left(M_{K}(R C)\right) \\
{[\mathrm{G} \& \mathrm{~S}]}\end{array}$ \\
\hline BE 39 & & -1.562 & 0.114 & -1.623 & 0.121 \\
NGC 752 & -1.541 & -1.544 & 0.114 & -1.566 & 0.116 \\
NGC 1817 & -1.485 & -1.660 & 0.114 & -1.749 & 0.180 \\
NGC 2099 & -2.063 & -2.068 & 0.114 & -2.143 & 0.182 \\
NGC 2204 & & -1.611 & 0.114 & -1.608 & 0.115 \\
NGC 2360 & & -1.144 & 0.114 & -1.159 & 0.121 \\
NGC 2420 & -1.685 & -1.666 & 0.114 & -1.681 & 0.115 \\
NGC 2477 & & -1.373 & 0.114 & -1.401 & 0.164 \\
NGC 2506 & & -1.582 & 0.114 & -1.596 & 0.106 \\
NGC 2527 & & -1.674 & 0.114 & -1.690 & 0.124 \\
NGC 2682 & -1.682 & -1.674 & 0.114 & -1.690 & 0.108 \\
NGC 6791 & & -1.385 & 0.114 & -1.482 & 0.132 \\
NGC 6819 & & -1.638 & 0.114 & -1.671 & 0.136 \\
\hline
\end{tabular}

Table 3. Cluster data.

\begin{tabular}{|c|c|c|c|c|c|c|c|}
\hline 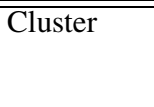 & $\begin{array}{c}E(B-V) \\
\text { mag } \\
(1)\end{array}$ & $\begin{array}{c}(m-M)_{V} \\
\text { mag } \\
(1)\end{array}$ & $\begin{array}{c}{[\mathrm{Fe} / \mathrm{H}]} \\
(2)\end{array}$ & $\sigma([\mathrm{Fe} / \mathrm{H}]$ & $\begin{array}{c}\log (\tau) \\
(3)\end{array}$ & $\begin{array}{c}M_{K}(R C) \\
\text { mag }\end{array}$ & $\begin{array}{c}\sigma\left(M_{K}(R C)\right) \\
\text { mag }\end{array}$ \\
\hline BE 39 & 0.11 & 13.50 & -0.15 & 0.09 & 9.90 & -1.56 & 0.11 \\
\hline IC 4651 & 0.11 & 10.25 & 0.00 & 0.09 & 9.06 & -1.81 & 0.11 \\
\hline MEL 66 & 0.14 & 13.95 & -0.38 & 0.06 & 9.44 & -1.70 & 0.11 \\
\hline NGC 188 & 0.09 & 11.35 & -0.12 & 0.06 & 9.63 & -1.36 & 0.11 \\
\hline NGC 752 & 0.04 & 8.35 & -0.09 & 0.06 & 9.05 & -1.54 & 0.11 \\
\hline NGC 1817 & 0.26 & 12.15 & -0.10 & 0.09 & 8.61 & -1.49 & 0.11 \\
\hline NGC 2099 & 0.27 & 11.55 & 0.27 & 0.15 & 8.54 & -2.06 & 0.11 \\
\hline NGC 2204 & 0.08 & 13.30 & -0.38 & 0.08 & 8.90 & -1.61 & 0.11 \\
\hline NGC 2243 & 0.06 & 13.45 & -0.48 & 0.06 & 9.03 & -1.87 & 0.11 \\
\hline NGC 2360 & 0.09 & 10.35 & -0.14 & 0.08 & 8.75 & -1.14 & 0.11 \\
\hline NGC 2420 & 0.05 & 12.10 & -0.44 & 0.06 & 9.05 & -1.69 & 0.11 \\
\hline NGC 2477 & 0.23 & 11.55 & 0.00 & 0.08 & 8.85 & -1.37 & 0.11 \\
\hline NGC 2506 & 0.05 & 12.60 & -0.42 & 0.09 & 9.05 & -1.58 & 0.11 \\
\hline NGC 2527 & 0.09 & 9.30 & 0.00 & 0.15 & 8.65 & -1.67 & 0.11 \\
\hline NGC 2682 & 0.04 & 9.80 & 0.02 & 0.06 & 9.41 & -1.68 & 0.11 \\
\hline NGC 3680 & 0.05 & 10.25 & -0.06 & 0.08 & 9.08 & -1.86 & 0.12 \\
\hline NGC 3960 & 0.29 & 12.15 & -0.27 & 0.09 & 8.82 & -1.08 & 0.11 \\
\hline NGC 5822 & 0.14 & 10.00 & 0.09 & 0.06 & 8.82 & -1.64 & 0.11 \\
\hline NGC 6134 & 0.35 & 11.10 & 0.29 & 0.15 & 8.97 & -1.02 & 0.11 \\
\hline NGC 6633 & 0.16 & 8.35 & -0.01 & 0.09 & 8.63 & -1.83 & 0.12 \\
\hline NGC 6791 & 0.15 & 13.40 & 0.40 & 0.06 & 9.64 & -1.39 & 0.11 \\
\hline NGC 6819 & $0.16^{* *}$ & $12.44^{* *}$ & 0.15 & 0.09 & 9.17 & -1.64 & 0.11 \\
\hline NGC 7789 & 0.27 & 12.45 & -0.13 & 0.08 & 9.23 & -1.52 & 0.11 \\
\hline TOM 2 & 0.30 & 15.60 & -0.45 & 0.06 & 9.01 & -1.67 & 0.11 \\
\hline $47 \mathrm{Tuc}^{*}$ & 0.044 & 13.45 & -0.700 & 0.070 & 10.08 & -1.34 & 0.21 \\
\hline NGC $362^{*}$ & 0.048 & 14.70 & -1.150 & 0.060 & 10.08 & -0.81 & 0.24 \\
\hline
\end{tabular}

References to data of the selected clusters: (1) Twarog et al. (1997), (2) Gratton (2000), (3) Chen et al. (2003), (*) Grocholski \& Sarajedini (2002), $(* *)$ Sarajedini (1999). The $M_{K}(R C)$ and the error resulting from this research can be found in the last two columns.

reddenings are used in both studies, error bars are determined only by the standard error about the mean in the box around the clump and the difference in both data releases. A mean difference of $0.03 \mathrm{mag}$ is found, meaning we found brighter RC's. Grocholski \& Sarajedini (2002) used the Second Incremental Data Release of the 2MASS project, while we use the All Sky Release. There are certainly some differences between both data releases. The All Sky Release offers more point sources in the same field, which can possibly cause a mean apparent magnitude of stars in that field to be more than 0.1 mag higher. To investigate this further we took data from the Second Data Release for the 13 clusters in common with Grocholski \& Sarajedini (2002) and used our data analysis protocol. There is good agreement with Grocholski \& Sarajedini (2002), as it should be. But we do find a mean difference of 0.02 mag between the All Sky and 2nd Release. This difference seems to be slightly dependent on distance. Because the same distance moduli and reddenings are used in both studies, error bars in this graph are determined only by the standard error about the mean in the box around the clump and the difference in both data releases. By using membership probabilities, mostly due to NGC 1817 , the difference between both investigations would enlarge it even more.

Grocholski \& Sarajedini (2002) conclude that for clusters having $-0.5 \leq[\mathrm{Fe} / \mathrm{H}] \leq 0.0$ and $10^{9.2} \leq \tau \leq 10^{9.9}$ years, one can simply use $\left\langle M_{K}(R C)\right\rangle=-1.61 \pm 0.04$ to determine distances. We tried to find a range in age and metallicity where we can assume a constant $M_{K}(R C)$ for all clusters with an as small error bar as possible. We scanned all ranges with $\Delta \log \tau>0.3$ 


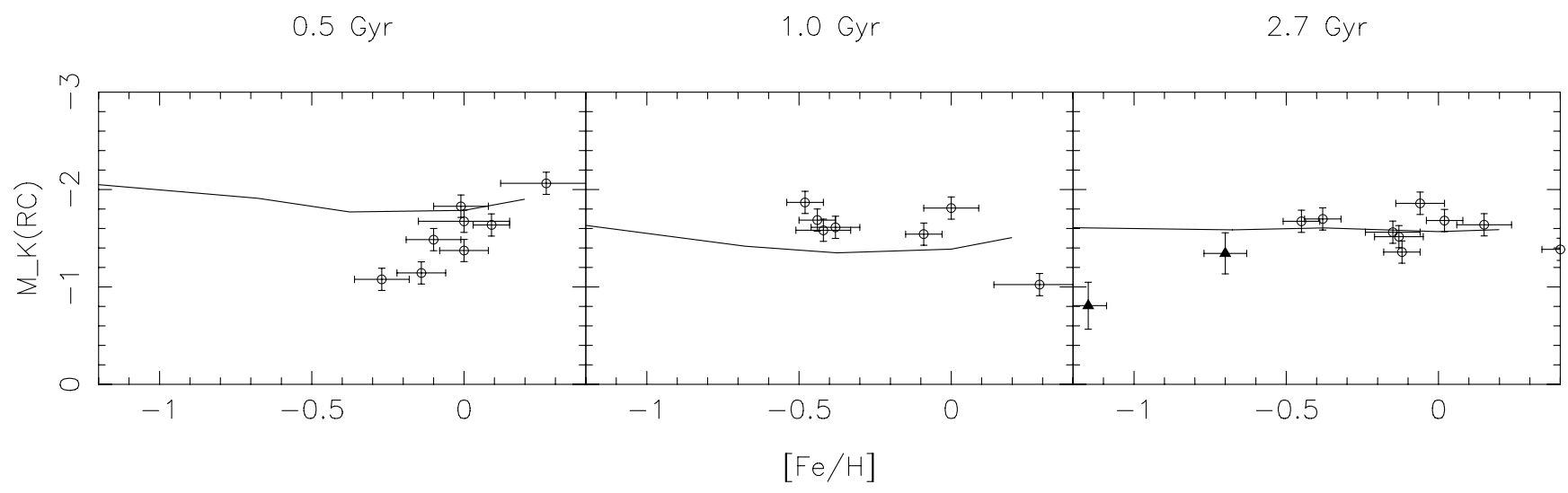

Fig. 6. Comparison of our results with the theoretical models of Salaris \& Girardi (2002). The two triangles represent the globular clusters.

$$
[\mathrm{Fe} / \mathrm{H}]=-0.2
$$$$
-0.1
$$

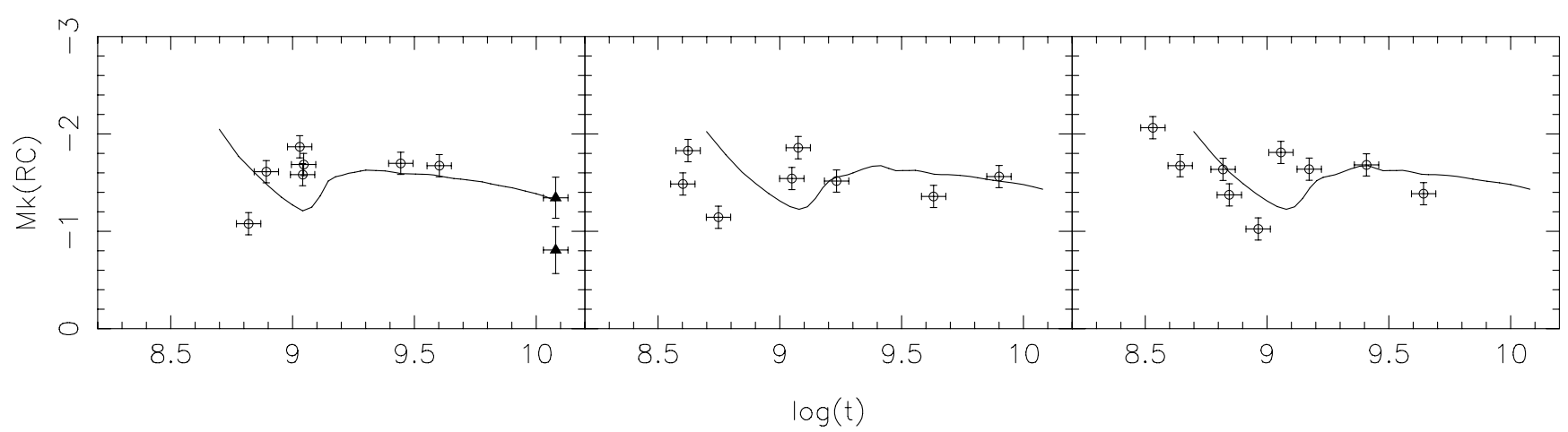

Fig. 7. Comparison of our results with the theoretical models of Salaris \& Girardi (2002). The two triangles represent the globular clusters.

and $\Delta[\mathrm{Fe} / \mathrm{H}]>0.3$ and with more than 5 clusters in it. We could not find one where the standard deviation of the mean, divided by the square of the amount of clusters in that range $\left(\frac{\sigma}{\sqrt{n}}\right)$, is less than \pm 0.05 , so we extend the range in age and metallicity of Grocholski \& Sarajedini (2002) and conclude that a constant value of $M_{K}(R C)=-1.57 \pm 0.05$ is a reasonable assumption to use for distance determinations for open clusters with a metallicity between -0.5 and +0.4 dex and an age between $10^{8.5}$ and $10^{9.9}$ years.

We compared our results with the theoretical models of Salaris \& Girardi (2002) for the $K$ band. Our results do not disagree with their theoretical predictions, whether regarding metallicity dependence, see Fig. 6, or in dependence on age, see Fig. 7. Each cluster is marked in these 3 graphs only once. The clusters were drawn in the graph that matches their age best in Fig. 6 or, respectively, metallicity in Fig. 7. As before, the globular clusters NGC 362 and 47 Tuc are represented by triangles. We would like to mention that these 2 globulars are not included in the calculation of our mean value for $M_{K}(R C)$, since they are not in the proper range of age and metallicity.

We conclude that within these limits of age and metallicity, a constant value of $M_{K}(R C)=-1.57 \pm 0.05$ mag can be adopted for each cluster. Since the rms of our sample is found to be $0.25 \mathrm{mag}$, this would result in an error of $12 \%$ on the distance.

\section{Discussion}

Although our results do not disagree with the theory of Salaris \& Girardi (2002), we still conclude that for the present observational dataset the dependence of $M_{K}(R C)$ can be neglected for metallicities between -0.5 and +0.4 dex and for ages between $10^{8.5}$ and $10^{9.9}$ years. We cannot make a statement outside these ranges. Two globular clusters do not seem to support the hypothesis of a constant value for $M_{K}(R C)$. More research is needed to draw conclusions for clusters with ages or metallicities outside these limits.

Our value of the mean red-clump magnitude in the $K$-band, $M_{K}(R C)$, is slightly higher than values in Alves (2000) and Grocholski \& Sarajedini (2002). The research of Alves (2000) states that the mean $M_{K}$ of the red clump stars in the solar neighborhood is $-1.61 \mathrm{mag}$. Grocholski \& Sarajedini (2002) find a value of $-1.62 \mathrm{mag}$ as the $\left\langle M_{K}(R C)\right\rangle$ of a sample of 14 open clusters. Although both values are close to each other, we claim that $-1.57 \mathrm{mag}$ is preferred for use in distance determinations. This is because we do not restrict ourselves to the solar neighborhood like Alves (2000) and use an updated dataset that is larger than the one of Grocholski \& Sarajedini (2002).

In closing, we would like to mention the Bologna Open Cluster Chemical Evolution Project, see Bragaglia \& Tosi (2006). This ongoing project will use a large sample of open clusters to investigate the chemical evolution of the Milky Way disk. The authors are still gathering data so as to analyze all clusters in a homogeneous way. In the near future we can expect a long list of clusters with homogeneous determinations of age, distance, reddening, and chemical abundances. We suggest that these data should be used not only for the original purpose, but also for further research of $M_{K}(R C)$ for open clusters.

Acknowledgements. This publication made use of data products from the Two Micron All Sky Survey, which is a joint project of the University of 
Massachusetts and the Infrared Processing and Analysis Center/California Institute of Technology, funded by the National Aeronautics and Space Administration and the National Science Foundation.

This research also made use of the WEBDA database, operated at the Institute for Astronomy of the University of Vienna.

Most important was the support of the best colleagues ever, so thank you Rachel Drummond, Clio Gielen, Bart Vandenbussche, Bram Acke, Pieter Deroo, Tijl Verhoelst, and Sofie Dehaes.

\section{References}

Alves, D. R. 2000, ApJ, 539, 732

Bessell, M. S., \& Brett, J. M. 1988, PASP, 100, 1134

Bragaglia, A., \& Tosi, M. 2006, AJ, 131, 1544

Cardelli, J. A., Clayton, G. C., \& Mathis, J. S. 1989, ApJ, 345, 245

Carpenter, J. M. 2001, AJ, 121, 2851

Chen, L., Hou, J. L., \& Wang, J. J. 2003, AJ, 125, 1397

Girardi, L., Bressan, A., Bertelli, G., \& Chiosi, C. 2000, A\&AS, 141, 371

Girardi, L., \& Salaris, M. 2001, MNRAS, 323, 109
Gratton, R. 2000, in Stellar Clusters and Associations: Convection, Rotation, and Dynamos, ed. R. Pallavicini, G. Micela, \& S. Sciortino, ASP Conf. Ser. 198, 225

Grocholski, A. J., \& Sarajedini, A. 2002, AJ, 123, 1603

Kubiak, M., McWilliam, A., Udalski, A., \& Gorski, K. 2002, Acta Astron., 52, 159

Paczyński, B., \& Stanek, K. Z. 1998, ApJ, 494, L219

Percival, S. M., \& Salaris, M. 2003, MNRAS, 343, 539

Piatti, A. E., Claria, J. J., \& Abadi, M. G. 1995, AJ, 110, 2813

Pietrzyński, G., Gieren, W., \& Udalski, A. 2003, AJ, 125, 2494

Salaris, M., \& Girardi, L. 2002, MNRAS, 337, 332

Sarajedini, A. 1999, AJ, 118, 2321

Stanek, K. Z., \& Garnavich, P. M. 1998, ApJ, 503, L131

Twarog, B. A., Ashman, K. M., \& Anthony-Twarog, B. J. 1997, AJ, 114, 2556

Udalski, A. 1998, Acta Astron., 48, 383

Udalski, A. 2000, ApJ, 531, L25

Van Helshoecht, V., \& Groenewegen, M. A. T. 2006, in ASP Conf. Ser., ed. C. Sterken, \& C. Aerts, 363

Zhao, G., Qiu, H. M., \& Mao, S. 2001, ApJ, 551, L85 
V. Van Helshoecht and M. A. T. Groenewegen: $M_{K}$ of the red clump as a distance indicator, Online Material $p 1$

\section{Online Material}


V. Van Helshoecht and M. A. T. Groenewegen: $M_{K}$ of the red clump as a distance indicator, Online Material $p 2$

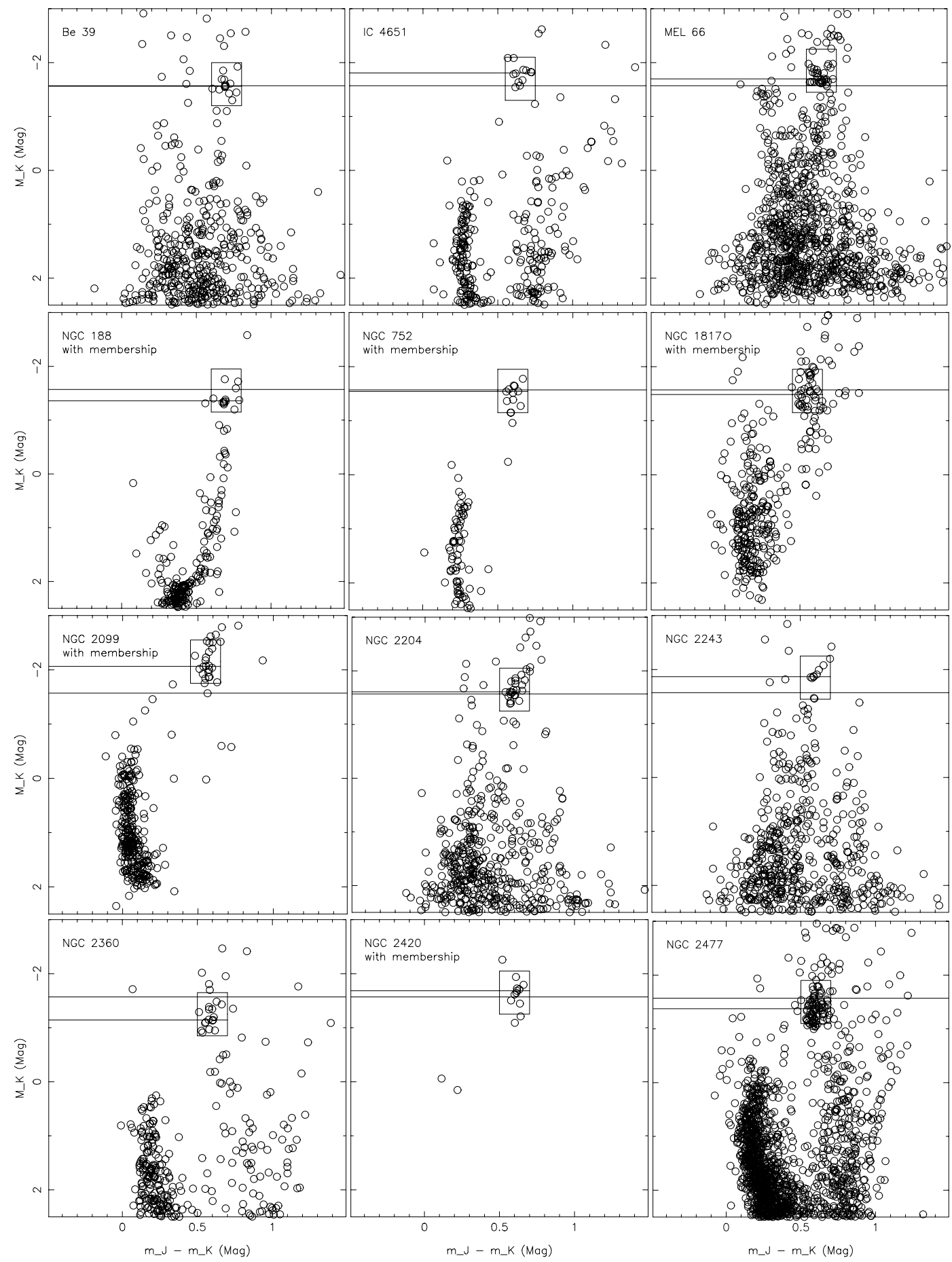

Fig. 8. CMD of every cluster in this investigation. The half line, coming out of the RC box, is drawn at the median $M_{K}(R C)$ of that particular cluster. The other line represents the mean $M_{K}(R C)$ of the entire cluster sample. 
V. Van Helshoecht and M. A. T. Groenewegen: $M_{K}$ of the red clump as a distance indicator, Online Material $p 3$

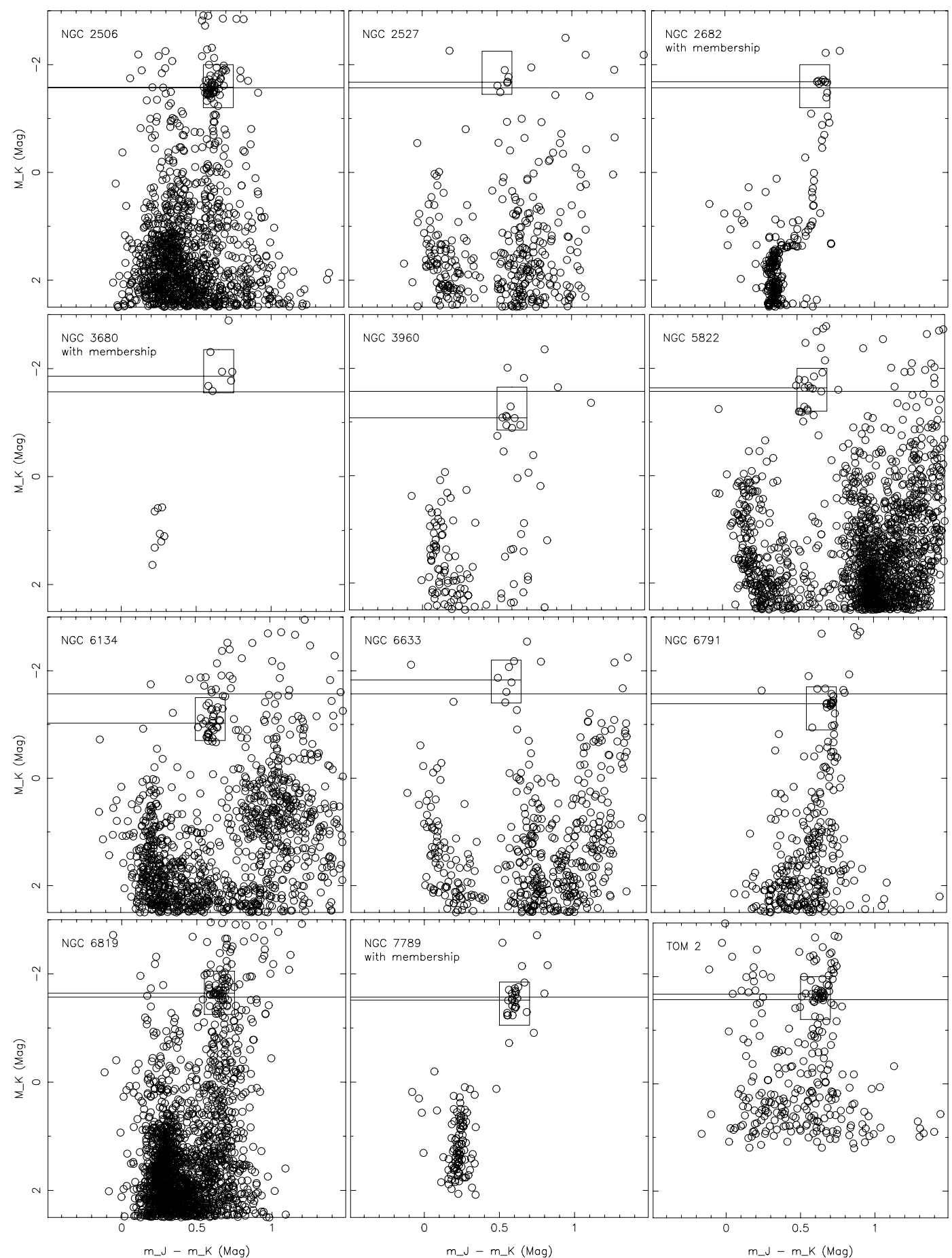

Fig. 9. CMD of every cluster in this investigation. The half line, coming out of the RC box, is drawn at the median $M_{K}(R C)$ of that particular cluster. The other line represents the mean $M_{K}(R C)$ of the entire cluster sample. 